\title{
Simultaneous relief of acute visceral and limb ischemia in complicated type $B$ aortic dissection by axillobifemoral bypass
}

\author{
Kyung Hwa Kim, MD, PhD, Jong Bum Choi, MD, PhD, and Ja Hong Kuh, MD, PhD
}

\begin{abstract}
A 78-year-old man with a history of aortic valve replacement and ascending aorta replacement sought treatment with severe back pain. Contrast-enhanced computed tomography revealed a Stanford type B acute aortic dissection (Figure 1). The patient was admitted to the intensive care unit, and antihypertensive drugs were administered. The next day, the patient had sharp pain in right lower extremity, pulse deficit in the right femoral artery, and abdominal pain with decreasing bowel sounds. Urgent computed tomography showed the progression of acute type $\mathrm{B}$ aortic dissection, which extended from the distal aortic arch to the bilateral external iliac arteries, and also demonstrated stenosis of the superior mesenteric artery and disappearance of flow in the right common iliac artery (Figure 2). We diagnosed malperfusion in acute type B dissection. The patient had became unstable and irritable. At first, we performed femorofemoral bypass; however, there was no flow in the bypass graft. Right axillobifemoral bypass grafting was therefore performed with an 8-mm ringed polytetrafluoroethylene graft tracked subcutaneously.

The next day, the patient's ischemic signs of right lower limb and abdominal pain were dramatically ameliorated, so the planned aortic fenestration and proximal stent-graft placement were cancelled. One year later, this patient is in good condition, with neither intermittent claudication nor abdominal angina. Follow-up computed tomographic angiograms at 1 and 12 months revealed the restoration of blood flow to the right iliac and mesenteric arteries with adequate aortic remodeling (Figure 3).
\end{abstract}

\footnotetext{
From the Department of Thoracic and Cardiovascular Surgery, Research Institute of Clinical Medicine, Chonbuk National University Medical School, Geumam-dong, South Korea.

Disclosures: Authors have nothing to disclose with regard to commercial support.

Received for publication June 20, 2013; accepted for publication June 27, 2013; available ahead of print April 21, 2013.

Address for reprints: Kyung Hwa Kim, MD, PhD, Department of Thoracic and Cardiovascular Surgery, Research Institute of Clinical Medicine, Chonbuk National University Medical School, 20 Geonji-Ro, Geumam-dong, Deokjin-gu, Jeonju, 561-180, South Korea (E-mail: tcskimgh@gmail.com).

J Thorac Cardiovasc Surg 2014;147:524-5

$0022-5223 / \$ 36.00$

Copyright (c) 2014 by The American Association for Thoracic Surgery

http://dx.doi.org/10.1016/j.jtcvs.2013.06.041
}

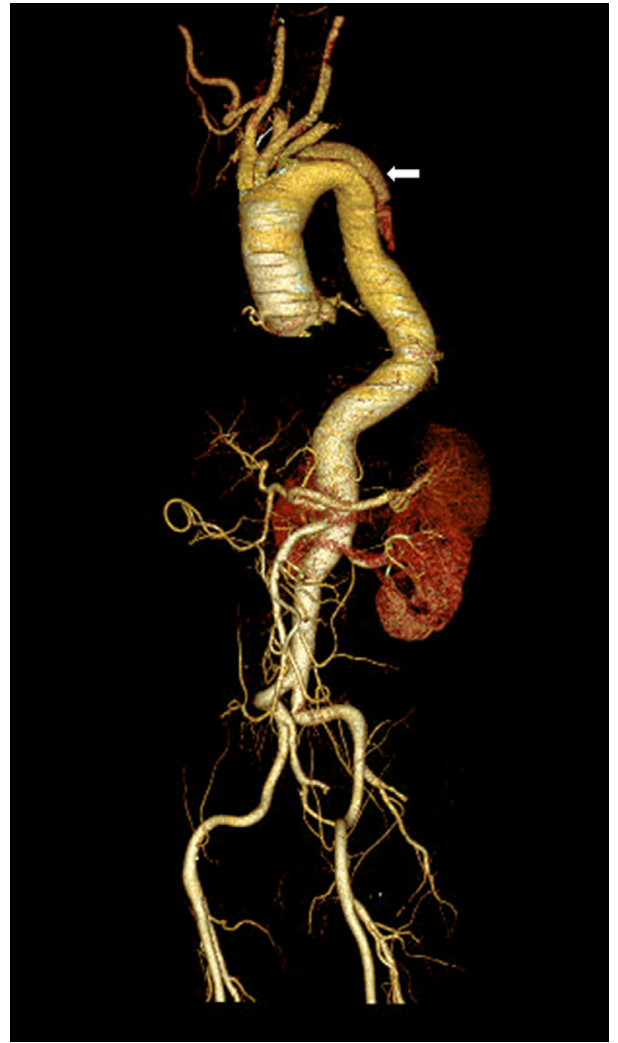

FIGURE 1. Contrast-enhanced computed tomography shows acute type B dissection (white arrow), originating distal to the left subclavian artery and extending only to the mid descending thoracic aorta. 


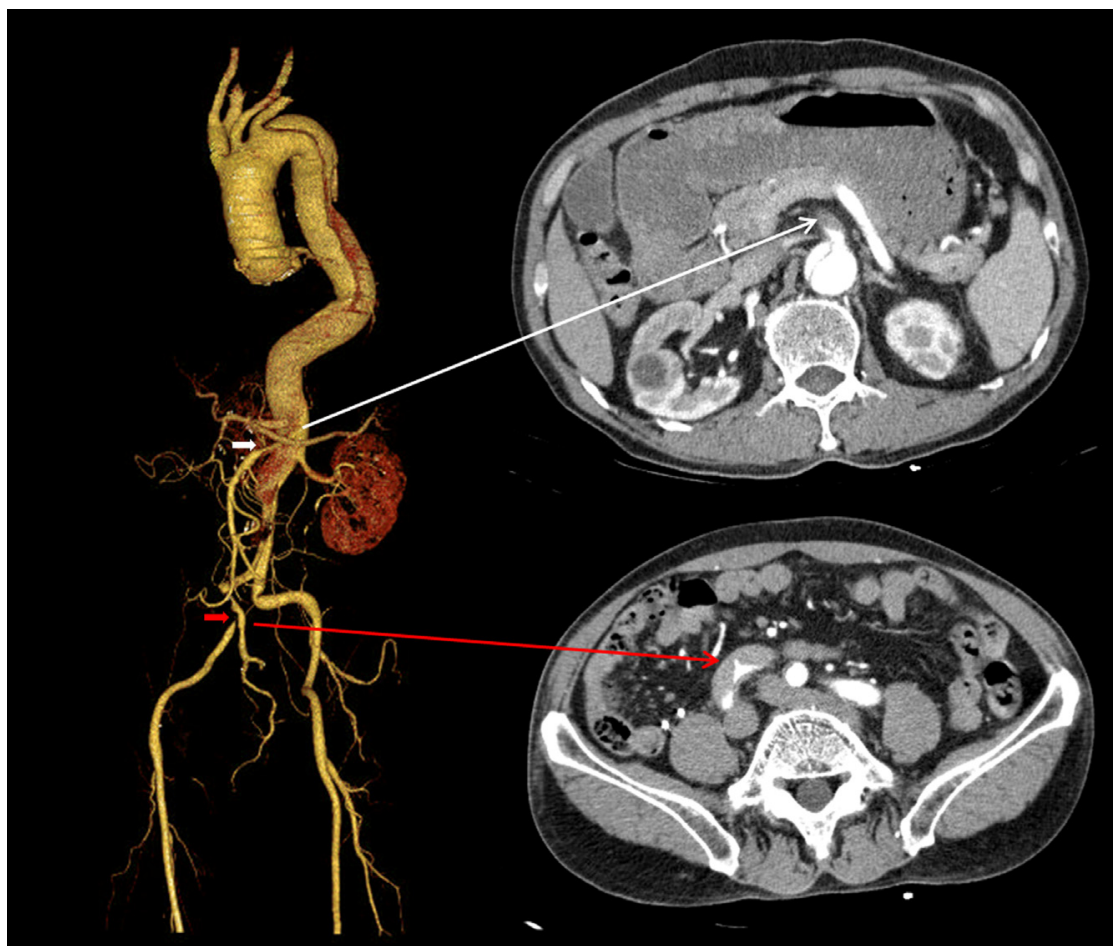

FIGURE 2. Urgent computed tomography shows the progression of acute type B aortic dissection, extending from the distal aortic arch to the bilateral external iliac arteries, and reveals the stenosis of superior mesenteric artery (white arrow) and disappearance of flow in the right common iliac artery (red arrow).

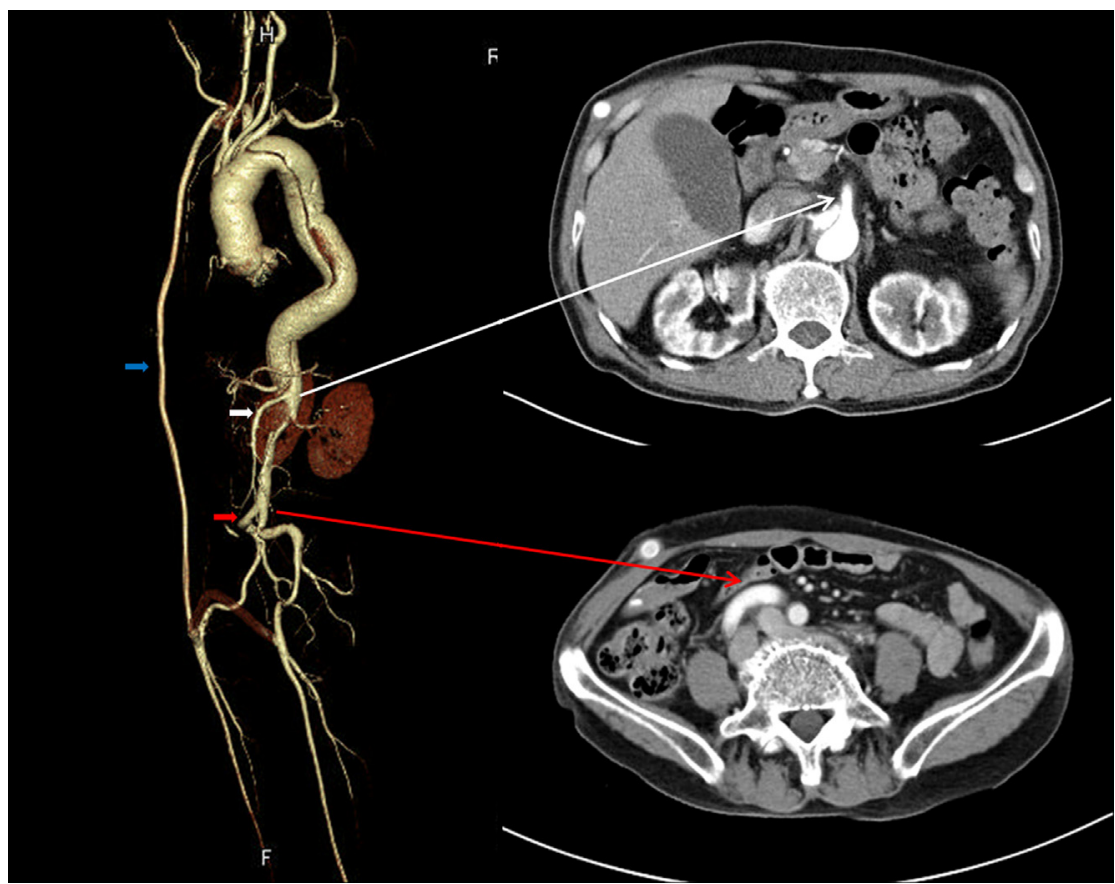

FIGURE 3. Follow-up computed tomography 12 months later shows the restoration of blood flow to the right iliac (white arrow) and mesenteric (red arrow) arteries with a patent vascular graft (blue arrow). 